(Aus der medizinischen Klinik in Heidelberg.)

\title{
Über \\ Verbrennung der Oxalsäure an Blutkohle und die Hemmung dieser Reaktion durch indifferente Narkotika.
}

Von

Otto Warburg.

(Mit 2 Textfiguren.)

Ordnet man die indifferenten Narkotika erstens aufsteigend nach ibren narkotischen Wirkungsstärken, zweitens aufsteigend nach ihren Teilungskoeffizienten ${ }^{1}$ ) zwischen $\ddot{O l}$ und Wasser, drittens aufsteigend nach ihrer Kapillaraktivität ${ }^{2}$ ), so erhält man ziemlich ähnliche Reihen. Narkotische Wirkungsstärke, Teilungsverhältnis $\frac{\ddot{O} l}{\text { Wasser }}$ und Kapillaraktivität sind also Grössen, die ziemlich parallel wachsen. Die Regel gilt nur in ganz roher Annäherung; es besteht nicht im entferntesten der Satz, dass Stoffe von gleicher narkotischer Wirkungsstärke gleiches Teilungsverhältnis oder gleiche Kapillaraktivität besitzen.

Der Parallelismus zwischen narkotischer Wirkungsstärke und Teilungsverhältnis $\frac{\text { Öl }}{\text { Wasser }}$ ist das Fundament der Lipoidtheorie; nach $H$. Meyer und Overton tritt dann Narkose ein, wenn die Konzentration des Narkotikums in den Zellipoiden einen gewissen

1) Hans Meyer, Schmiedeberg's Arcb. Bd. 42 S. 109; und Baum, Schmiedeberg's Arch. Bd. 42 S. 119 und Bd. 46 S. 338. - Overton, Studien über die Narkose. Jena 1901.

2) J. Traube, Pflüger's Arch. Bd. 140 S. 109. 1911; hier sind auch frühere Arbeiten Traube's zitiert; ferner Traube, Pflüger's Archiv Bd. 153 S. 276. 1913 . 
Betrag erreicht hat. Demgegenüber vertritt J. Traube seit Jahren die Auffassung, dass die Wirkung der Narkotika nicht auf ihrer Lipoidlöslichkeit, sondern auf ihrer Kapillaraktivität beruhe. -

Wie trüher gezeigt wurde ${ }^{1}$ ), verlangsamen die indifferenten Narkotika die Oxydationsgeschwindigkeit sauerstoffatmender Zellen; die Konzentrationen, die zur Oxydationshemmung erforderlich sind, liegen erheblich höher als die zur Gehirnnarkose erforderlichen; die Reihenfolge der nach ihren Wirkungsstärken geordneten Stoffe ist für Oxydationshemmung und Gehirnnarkose die gleiche. Auch die oxydationshemmenden Wirkungsstärken wachsen also mit dem Teilungsverhältnis $\frac{\ddot{O} l}{\text { Wasser }}$ und mit der Kapillaraktivität.

Solange wir weiter nichts wissen, als dass die Wirkungsstärken in einem gewissen Parallelismus zu den angeführten Eigenschaften stehen, wird eine noch so häufige Diskussion nicht entscheiden, ob der springende Punkt die Lipoidlöslichkeit, die Kapillaraktivität oder möglicherweise eine Eigenschaft ist, auf die bisher die Aufmerksamkeit noch nicht gelenkt wurde. In der Tat wissen wir heute hinsichtlich der Gehirnnarkose nichts, was eine Entscheidung ermöglichte.

Anders steht es mit der Verlangsamung der Oxydationsgeschwindigkeit. Ursprünglich auf dem Boden der Lipoidtheorie stehend ${ }^{2}$ ), wurde ich bald durch eine Reihe von Beobachtungen zweifelhaft und schlug deshalb vor ${ }^{3}$ ), die Entscheidung zugunsten oder ungunsten der Lipoidtheorie zu vertagen. Seitdem ist der Mechanismus der Oxydationshemmungen bis zu einem gewissen Grad verständlich geworden ${ }^{4}$ ). Wir wissen heute, dass unter dem Einfuss der Narkotika die Fermente ausgeflockt oder ihre aktiven Oberflächen verkleinert werden. Wir wissen weiterhin, dass die Narkotika sich an den Verbrennungsorten der Zelle anreichern, worauf höchstwahrscheinlich die stärkere Wirkung der Narkotika auf Fermentreaktionen innerhalb der Zelle beruht. Wir wissen endlich,

1) 0. Warburg, Hoppe-Seyler's Zeitschr. f. physiol. Chemie Bd. 69 S. 452. 1910. Mit späteren Arbeiten zusammengefasst in Asher-Spiro, Ergebnisse Bd. 14.

2) Hoppe-Seyler's Zeitschr. f. physiol. Chemie Bd. 69 S. 452.

3) Pfl üger's Arch. Bd. 144 S. 465.

4) 0. Warburg, Asher-Spiro, Ergebn. d. Physiol. l. c. 
für einige Narkotika wenigstens, dass eine Anreicherung auch nach Entfernung der Lipoide stattfindet: alles Tatsachen, die zur Lipoidtheorie nur unter Aufstellung weiterér Hypothesen passen, auf Grund der Tra u be'schen Auffassung jedoch zwanglos erklärt werden können.

Ich stehe heute auf dem Standpunkt, dass nicht die Lipoidlöslichkeit, sondern die Kapillaraktivität diejenige Eigenschaft ist, die die oxydationshemmende Wirkung der Narkotika bedingt. Die Verbrennungen in sauerstoffatmenden Zellen sind Oxydationskatalysen an Oberflächen und werden durch indifferente Narkotika gehemmt, weil sich diese Stoffe ã̃ den Oberflächen anreichern ${ }^{1}$ ) und hier das Adsorptionsmilieu verändern.

Im folgenden soll nun ein Modell beschrieben werden, an dem sich demonstrieren lässt, wie indifferente Narkotika chemische Umsatzgeschwindigkeiten verlangsamen auf Grund ihrer Eigenschaft, an Oberflächen zu gehen. Es wird gezeigt werden, dass $0 \times a l-$ säure bei $38^{\circ}$ an der Oberfläche von Blutkohle zu Koblensäure und Wasser verbrennt, und dass die Geschwindigkeit dieser Reaktion in ähnlicher Weise durch indifferente Narkotika verlangsamt wird, wje die Oxydationsgeschwindigkeit in Zellen.

Daneben wurden auch andere Oxydationskatalysen auf ibr Verhalten gegenüber indifferenten Narkotika geprüft, besonders auch die Oxydationsbeschleunigung des Lezithins durch Eisensalz, die von Thunberg ${ }^{2}$ ) zuerst beobachtet wurde und nach neueren Feststellungen im Mechanismus sauerstoffatmender Zellen eine Rolle spielt $\left.{ }^{3}\right)$. Eine Beeinflussung der Geschwindigkeit dieser Reaktion durch indifferente Narkotika in Konzentrationen, wie sie biologisch in Betracht kommen, konnte nicht festgestellt werden. Auch dieses negative Resultat ist im Zusammenhang mit unseren obigen Ausführungen bemerkenswert; gehört doch Lezithin zu den wesentlichsten Bestandteilen der "Zellipoide".

1) Diese Oberflächen können natürlich aus verschiedenem Material, Eiweiss, Nukleoproteiden, Lipoidev oder anderen Stoffen bestehen; darüber lässt sich heute mit Gewissheit nichts aussagen.

2) Skandinav. Arch. Bd. 24 S. 90.1910.

3) 0 . Warburg und O. Meyerh of in Asher-Spiro, Ergebnisse, 1. c. 


\section{Die Verbrennung der Oxalsäure an Blutkohle.}

Dass fein verteilte Kohle Oxydationen beschleunigt, ist schon seit langer Zeit bekannt. Zur Demonstration in der Vorlesung empfahl A. W. v. Hof $\mathrm{man} \mathbf{n}^{1}$ ), eine alkoholische Lösung von Leukanilin mit Kohle aufzukochen, wobei sofort die rote Farbe des Rosanilins auftritt.

Was die Oxalsäure anbetrifft, so teilte Freundlich²) die Beobachtung mit, dass beim Schütteln einer wässrigen Säurelösung mit Kohle dauernd Säure aus der Lösung verschwindet. Freund lich knüpfte an diesen Befund die Vermutung, dass die Oxalsäure an der Kobleoberfläche "durch eine chemische Umsetzung" zerstört würde.

Zunächst konnte ich feststellen, dass mit Oxalsäure beladene Kohle Sauerstoff verbraucht. Verschiedene Kohlesorten, mit gleichen Oxalsäurekonzentrationen im Gleichgewicht, verbrauchten sehr verschiedene Mengen Sauerstoff; am meisten verbrauchte die Mercksche Blutkohle, weniger die Kahlbaum'sche Blutkohle. Nicht nachweisbar war eine Sauerstoffzehrung bei Verwendung Kahlbaum'scher Kohle aus Rohrzucker. In der gleichen Reihenfolge standen die verschiedenen Kohlepräparate hinsichtlich ihrer Fähigkeit, Oxalsäure zu adsorbieren; wurden gleiche Mengen Kohle zu gleichen Volumina gleichkonzentrierter Oxalsäure gegeben und nach 2 Minuten langem Schütteln die Oxalsäurekonzentrationen in der Lösung durch Titrieren gemessen, so zeigte sich, dass die Rohrzuckerkohle fast nichts, die Kahlbaum' sche Blutkohle mehr, die Mercksche Blutkohle bedeutend mehr Säure aus der Lösung fortgenommen hatte. Zu allen im folgenden beschriebenen Versuchen wurde die Merck'sche Blutkoble (puriss. mit Säure gereinigt) verwendet. Das Oxalsäurepräparat war von Kahlbaum; die angegebenen Gewichtsprozente beziehen sich auf die wasserfreie Verbindung $\mathrm{C}_{2} \mathrm{H}_{2} \mathrm{O}_{4}$.

Um für die Stärke der Adsorption und für die Oxydationsgeschwindigkeit einen Anhaltspunkt zu geben, seien folgende Zahlen angeführt: an $1 \mathrm{~g}$ Blutkohle waren, jm Gleichgewicht mit einer $0,071 \%$ igen wässrigen Oxalsäurelösung, ca. $50 \mathrm{mg}$ Oxalsäure (in der Ausdrucksweise Freundlich's: bei einem $c$ von 0,008 Molen

1) Berliner Berichte Bd. 7 S. 530.1874.

2) Kapillarchemie S. 163. Leipzig 1909. 
pro Liter betrug das $\frac{x}{m} 0,56$ Millimole). $1 \mathrm{~g}$ Blutkohle, im Gleichgewicht mit einer $0,071 \%$ igen Oxalsäurelösung, verbrauchte bei $38^{\circ}$ in der ersten Stunde etwa 1,1 ccm Sauerstoff; oder $50 \mathrm{mg}$ Oxalsäure, die sich an $1 \mathrm{~g}$ Kohle befanden, verbrauchten in der ersten Stunde etwa 1,1 cem Sauerstoff.

Die Versuche waren so angeordnet, dass stets $90 \mathrm{mg}$ Kohle durch Waschen auf der Zentrifuge mit einer bekannten Oxalsäurekonzentration annähernd in Gleichgewicht gebracht wurden. Bei einer Konzentration von 0,008 Molen genügte dreimaliges Waschen mit $90 \mathrm{ccm}$ Lösung. Dann wurde in ein kleines graduiertes Zentrifugierglas übergespült, wieder zentrifugiert, die überstebende Flüssigkeit bis auf $1 \mathrm{ccm}$ abgehebert, die Kohle aufgewirbelt, die Suspension in das später beschriebene Bestimmungsgläschen gegossen und das Zentrifugierglas mit $0,5 \mathrm{ccm}$ Oxalsäurelösung nachgespült. Die $0,5 \mathrm{ccm}$ Spülflüssigkeit kamen gleichfalls in das Bestimmungsgläschen, das also dann $90 \mathrm{mg}$ Kohle in $1,5 \mathrm{ccm}$ Flüssigkeit enthielt oder $1,5 \mathrm{ccm}$ einer $6 \%$ igen Kohlesuspension. Das Bestimmungsgläsehen wurde mit dem Manometer verbunden und in den Thermostaten bei ca. $38^{\circ}$ gehängt; zunächst wurde bei offenem Hahn 10 Minuten geschüttelt, bis Temperaturgleichgewicht eingetreten war und sich die Kohlesuspension mit den Gasen der Luft bei der Versuchstemperatur in Gleichgewicht gesetzt hatte. Dann wurden die Hähne geschlossen und die Sauerstoffabsorptionen unter beständigem Schütteln gemessen.

Ich habe anfangs Bedenken gehabt, ob sich mit Kohle, die bekanntlich grosse Gasmengen aufnehmen und abgeben kann, genaue gasanalytische Versuche anstellen lassen. Diese Bedenken waren unbegründet. Das liess sich feststellen durch Kontrollen, in denen mit Wasser gewaschene Kohle (je $90 \mathrm{mg} \mathrm{Kohle} \mathrm{dreimal} \mathrm{mit} 90 \mathrm{ccm}$ Wasser) in gleicher Weise auf Sauerstoffzehrung geprüft wurde. Derartige Kohle gab regelmässig beim Schütteln im Thermostaten eine geringe Druckverminderung, die jedoch gegen die Zehrung der mit Oxalsäure beladenen Kohle nicht in Betracht kam $^{1}$ ).

1) Kohle, die nicht in der angegebenen Weise mit Wasser gewaschen, sondern in wenig Flüssigkeit suspendiert, eingehängt wurde, zeigte eine etwas stärkere Gasaufnahme. In der Tat wird durch das Waschen mit luftgesättigten Flüssigkeiten die Kohle mit den Gasen der Luft in Gleichgewicht gesetzt. Bei den hier beschriebenen Versuchen wurde stets, a ch bei allen Kontrollen, mindestens dreimal mit $90 \mathrm{ccm}$ gewaschen, bei Verwendung von $90 \mathrm{mg}$ Kohle. 
Oxydationsgeschwindigkeit und Konzentration.

Setzt man Kohle mit verschiedenen Oxalsäurekonzentrationen in Gleichgewicht, so stösst man auf die merkwürdige Tatsache, dass, von einer gewissen Grenze an, die Oxydationsgeschwindigkeit mit steigenden Oxalsäurekonzentrationen sinkt. In der nachstehenden graphischen Darstellung sind auf der Abszisse die Zeiten in Minuten, auf der Ordinate die verbrauchten Sauerstoffmengen in Kubikmillimetern

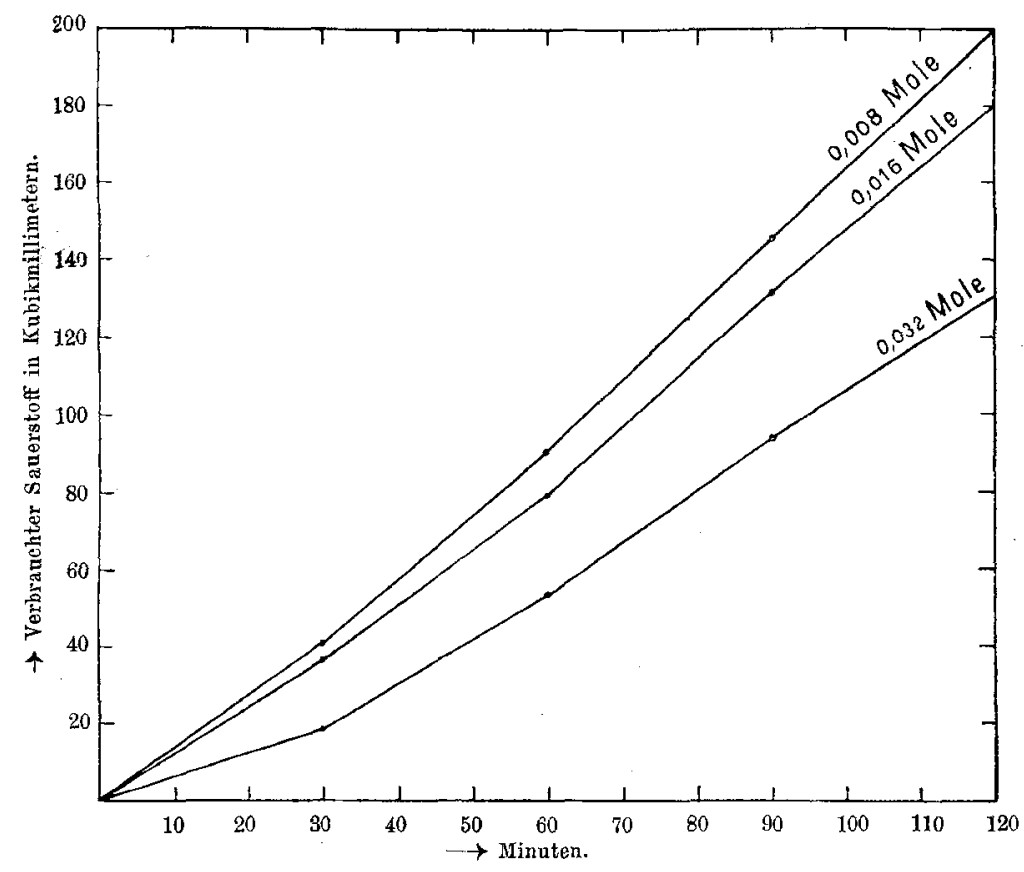

Fig. 1. Sauerstoffverbrauch bei verschiedenen Oxalsäurekonzentrationen. $38^{\circ}$.

eingetragen. Man sieht, dass der Sauerstoffverbrauch der Oxalsäurekohle nicht linear mit der Zeit wächst, sondern dass die Oxydationsgeschwindigkeit im Laufe der ersten Stunde anwächst. Das wurde ganz regelmässig in mehr als 50 Versuchen beobachtet. Man sieht weiterhin, dass bei einer Konzentration von 0,008 Molen (pro Liter) die Oxydationsgeschwindigkeit beträchtlich höher ist als bei einer Konzentration von 0,016 und von 0,032 Molen. Dieses Verhalten wurde verfolgt bis zu einer Konzentration von 0,8 Molen pro Liter und eine beständige Abnahme der Oxydationsgeschwindigkeit beobachtet. Eine Erklärung dieses Phänomens kann ich nicht geben; doet sei daran erinnert, dass in einer Oxalsäurelösung ausser der un. 
dissoziierten Oxalsäure noch Wasserstoffionen und zwei Arten von Anionen vorkommen, dass das Verhältnis dieser vier Körper bei verschiedenen Konzentrationen ein verschiedenes ist, und dass möglicherweise gegenseitige Adsorptionsverdrängungen eine Rolle spielen.

Der Temperaturkoeffizient.

Je $90 \mathrm{mg}$ Kohle, die im Gleichgewicht waren mit einer Oxalsäurekonzentration von 0,008 Molen, wurden in $6 \%$ iger Suspension bei $37,5^{\circ}$ und bei $15,5^{\circ} 80$ Minuten lang geschüttelt. Bei $37,5^{\circ}$ waren nach dieser Zeit verbraucht: $0,120 \mathrm{cem}$ Sauerstofi; bei $15,5^{\circ}$; $0,023 \mathrm{ccm}$ Sauerstoff. Für die Temperaturdifferenz von $22^{\circ}$ ist also das Verhältnis der Geschwindigkeiten 5,2, woraus sich für das Intervall von $10^{\circ}$ ein Koeffizient von 2,1 berechnet.

Dieser Wert ist nur ein Annäherungswert, weil, wie oben erwähnt, die Geschwindigkeiten bei ein und derselben Temperatur nicht ganz konstant sind und aus den Kurven auch keine Konstanten berechnet wurden, mitbin die Vorbedingung für eine genaue Bestimmung eines Temperaturkoeffizienten nicht gegeben war. Soweit aber dürfte die angegebene Zahl zu verwerten sein, dass die Diffusion als gesehwindigkeitsbestimmendes Moment hier ausgesehlossen werden kann. Es ist das übrigens leicht verständlich, denn die Hauptmenge der Oxalsäure, die in dem System vorhanden ist, befindet sich ja, nach den Adsorptionsmessungen, von Anfang an an der Kohle (von den $5,6 \mathrm{mg}$ Säure, die in $1,5 \mathrm{ccm}$ der $6 \%$ igen Kohlesuspension enthalten sind, befinden sich am Anfang des Versuchs $4,5 \mathrm{mg}$ an der Kohle!).

Die Gleichung der Verbrennung.

In dem Maasse, als Sauerstoff absorbiert wird, entwickelt sich bei der Reaktion Kohlensäure. Wurden $90 \mathrm{mg}$ Kohle, die mit einer Konzentration von 0,008 Molen auf die beschriebene Art in Gleichgewicht gebracht waren, in $6 \%$ iger Suspension bei $38^{\circ} 1$ Stunde geschüttelt, so waren beispielsweise $0,103 \mathrm{ccm}$ Sauerstoff absorbiert und $0,392 \mathrm{~cm}$ Kohlensäure neu gebildet (Methodik siehe unten). Es sind das annähernd 4 Moleküle Kohlensäure auf 1 Molekül Sauerstoff (berechnet für 4 Moleküle $0,412 \mathrm{ccm}$ Kohlensäure; die Differenz fallt in die Fehlergrenzen). Auf Grund dieses Resultates ist für die Verbrennung folgende Formel aufzustellen: 


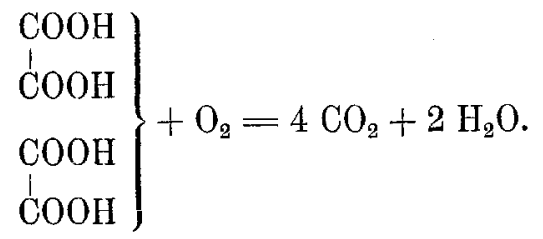

\section{Die Hemmung der Oxalsäureverbrennung durch Urethane.}

Die Versuche waren so angeordnet, dass in vier Zentrifugiergläser je $90 \mathrm{mg}$ Kohle gegeben wurde: in einem Glase wurde mit 0,008 molarer Oxalsäurelösung gewaschen, in den anderen drei Gläsern mit 0,008 molaren Oxalsäurelösungen, denen die zu prüfenden Urethane in verschiedenen Mengen zugesetzt waren. Im übrigen wurde weiter verfahren, wie unter I. beschrieben, also die Kohle schliesslich in 1,5 cem Flüssigkeit suspendiert, im Thermostaten bei $38^{\circ}$ geschüttelt.

Zur Prüfung, ob beim Waschen mit den verschiedenen Stoffen Gleichgewicht annähernd erreicht war, bediente ich mich des Tra ube'schen Stalagmometers ${ }^{1}$ ) und wusch die Kohle so lange, bis die Tropfenzahl des von der Kohle abzentrifugierten Waschwassers mit der Tropfenzahl der zu prüfenden Lösung übereinstimmte. Bei Phenylurethan - von den geprüften Substanzen diejenige, die am stärksten adsorbiert wird - war nach viermaligem Waschen mit $90 \mathrm{ccm}$ Gleichgewicht erreicht, wenn die Konzentration $0,05 \%$ betrug. Sollte von Phenylurethan die Konzentration $0,005 \%$ geprüf werden, so wurde sechsmal mit $90 \mathrm{ccm}$ gewaschen und angenommen, dass Gleichgewicht erreicht war. Mit Hilfe des Stalagmometers konnte das nicht mehr festgestellt werden, weil die Erniedrigung der Oberflächenspannung durch eine so kleine Menge Phenylurethan zu klein ist, um eine beträchtliche Zunahme der Tropfenzahl zu verursachen. Möglicherweise also war in diesem Fall noch kein Gleichgewicht erreicht. Weniger als dreimal mit $90 \mathrm{~cm}$ wurde in keinem Fall gewaschen.

Die Hemmungen sind bei dieser Versuchsanordnung bestimmt b e $\mathrm{i}$ konstanter Oxalsäure-und bei konstanter Sauerstoffkonzentration ${ }^{2}$ ) in der Suspensionsflüssigkeit, indem die Oxal-

1) J. Traube in Abderhalden's Biochem. Arbeitsmethoden 1912 S. 1857.

2) Genauer: "Anfangskonzentration", da die Konzentrationen im Laufe des Versuches abnehmen. 
säurekonzentration 0,008 molar, die Sauerstoffkonzentration durch die Sättigungskonzentration der Flüssigkeit mit Luft bei $38^{\circ}$ gegeben war.

Wenn man sich die Aufgabe stellt, die Verhältnisse des biologischen Versuches möglichst nachzuahmen, so ist diese Anordnung bezüglich des Sauerstoffs sicher richtig. Denn wenn die Oxydations-

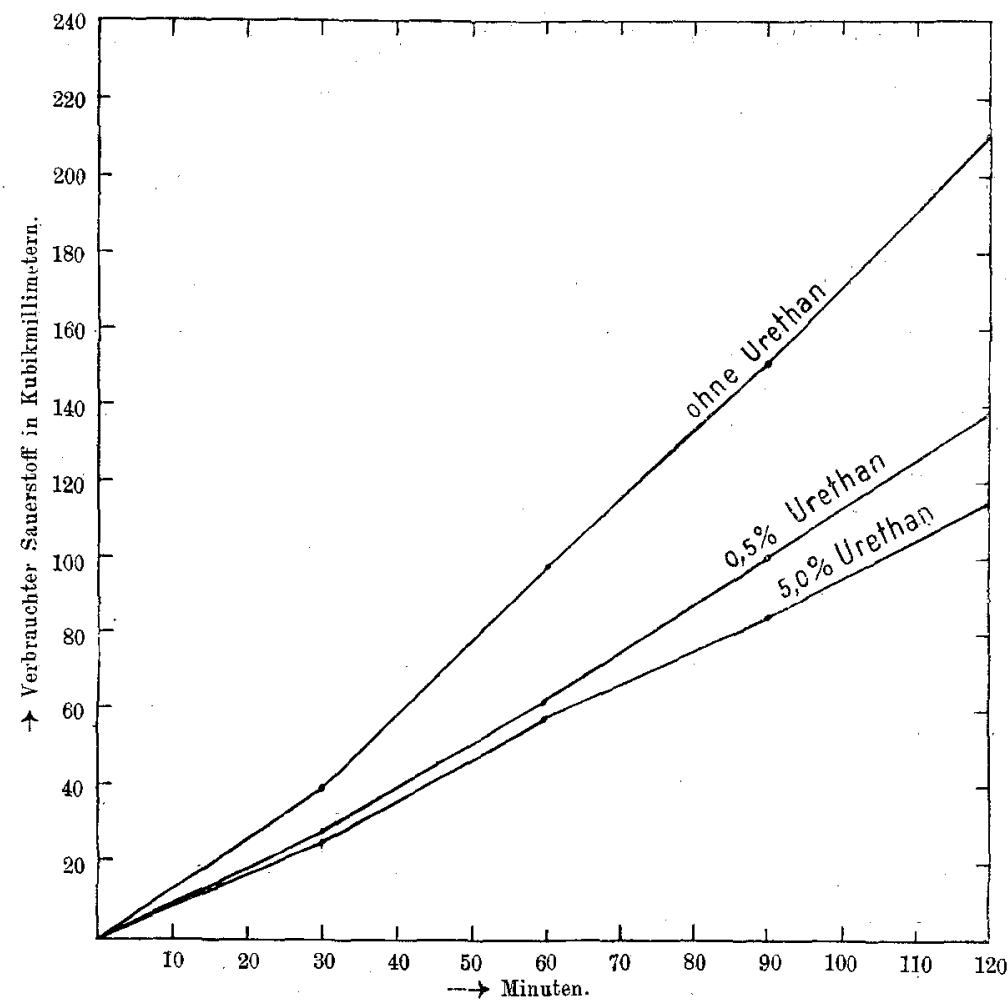

Fig. 2. Hemmung durch Methylurethan. $38^{\circ}$.

geschwindigkeit in Zellen mit und ohne Urethan verglichen wurde, so waren stets die Sauerstoff-Aussenkonzentrationen zu Beginn des Versuches die gleichen. Bezüglich der anderen Komponente des Systems, der Oxalsäure, könnte man im Zweifel sein, ob man den in der Zelle gegebenen Verhältnissen näher komınt, wenn man die Oxalsäurekouzentration oder die Oxalsäuremenge konstant hält. Ich habe Versuche angestellt auch bei konstanter Oxalsäure menge; zu 1,5 ccm $6 \%$ iger Kohlesuspensionen, die mit verschiedenen Urethankonzentrationen in Gleichgewicht gebracht waren, wurden gleiche 
Mengen Oxalsäure zugesetzt. Infolge von Adsorptionsverdrängung waren dann die Oxalsäurekonzentrationen in der Suspensionsflüssigkeit verschieden. Die Verhältnisse werden jedoch bei einer solchen Anordnung etwas kompliziert, und ich beschränke mich deshalb auf Wiedergabe der Versuche mit konstanter Oxalsäurekonzentration.

Der typische Verlauf eines derartigen Hemmungsversuches ist in der vorstehenden graphischen Darstellung dargestellt (Fig. 2). Wir sehen zunäcbst wieder in der Kontrolle ohne Urethan, dass die Oxydationsgeschwindigkeit anfangs etwas zunimmt; auch bei $0,5 \%$ Urethan ist diese Zunahme deutlich, weniger bei $5 \%$ Urethan. Die Folge davon ist, dass die Hemmungen bei höheren Urethankonzentrationen etwas progressiv sind, das heisst: die Hemmungen sind in den Anfangsperioden etwas kleiner als später. Immerhin macht das nicht viel aus. Um die durch die Progression bedingte Unregelmässigkeit nach Möglichkeit auszuschalten, habe ich zum Vergleich die nach einer bestimmten Zeit beobachteten Hemmungen ausgerechnet und in der folgenden Zusammenstellung die Hemmungen nach zweistündiger Dauer des Versuches angegeben (ist ohne Urethan nach 2 Stunden die Sauerstoffmenge $a$ verbraucht, bei Gegenwart von Urethan die Sauerstoffmenge $b$, so ist die Oxydationshemmung $\frac{a-b}{a}$ oder, in Prozenten ausgedrückt, $\frac{a-b}{a} \times 100$ ).

(Siehe Tabelle I und II auf S. 557.)

Aus der Zusammenstellung geht unzweideutig -hervor, dass Methylurethan schwächer wirkt als Äthylurethan, dieses schwächer als Propylurethan, dieses sehwächer als Phenylurethan. Wir haben also dieselbe Reihenfolge der Wirkungsstärken, wje sie für die Oxydationshemmungen in lebenden Zellen gefunden wurden.

Aus einem Vergleich der Tabelle I mit Tabelle II geht ferner hervor, dass die Konzentrationen, die eine bestimmte Oxydationshemmung in Zellen ${ }^{1}$ ) bewirken, vielfach die Oxydationsgeschwindigkeit unseres Modells um einen ähnlichen Betrag vermindern. Vielleicht ist diese Übereinstimmung mehr als ein Zufall, nicht durch die

1) Die Konzentrationen für Blutzellen und andere Zellen sind nicht sehr| verschieden. Vgl, O. Warburg in Asher-Spiro, Ergebnisse 1. c. 
Tabelle I.

0xalsäure-Kohle.

\begin{tabular}{|c|c|c|}
\hline Substanz & $\begin{array}{l}\text { Gewichts- } \\
\text { prozente }\end{array}$ & $\begin{array}{l}\text { Prozentische } \\
\text { Oxydations- } \\
\text { hemmung } \\
\left(\frac{a-b}{a} \times 100\right)\end{array}$ \\
\hline $\begin{array}{l}\text { Methyl- } \\
\text { urethan }\end{array}$ & $\begin{array}{c}0,05 \\
0,5 \\
5,0 \\
10,0\end{array}$ & $\begin{array}{r}0 \\
34 \\
46 \\
60\end{array}$ \\
\hline $\begin{array}{l}\text { Äthyl- } \\
\text { urethan }\end{array}$ & $\begin{array}{r}0,5 \\
5,0 \\
10,0\end{array}$ & $\begin{array}{l}42 \\
65 \\
76\end{array}$ \\
\hline $\begin{array}{l}\text { Propyl- } \\
\text { urethan. }\end{array}$ & $\begin{array}{l}0,05 \\
0,5 \\
5,0\end{array}$ & $\begin{array}{l}41 \\
72 \\
92\end{array}$ \\
\hline $\begin{array}{l}\text { Phenyl- } \\
\text { urethan }\end{array}$ & $\begin{array}{l}0,005 \\
0,05\end{array}$ & $\begin{array}{l}34 \\
90\end{array}$ \\
\hline
\end{tabular}

Tabelle II.

Rote Blutzellen ${ }^{1}$ ).

\begin{tabular}{|c|c|c|}
\hline Substanz & $\begin{array}{l}\text { Gewichts- } \\
\text { prozente }\end{array}$ & $\begin{array}{l}\text { Prozentische } \\
\text { Oxydations- } \\
\text { hemmung } \\
\left(\frac{a-b}{a} \times 100\right)\end{array}$ \\
\hline $\begin{array}{l}\text { Methyl- } \\
\text { urethan }\end{array}$ & 10 & ca. 60 \\
\hline $\begin{array}{l}\text { Äthyl- } \\
\text { urethan }\end{array}$ & $\begin{array}{l}1,25 \\
2,5 \\
5,0\end{array}$ & $\begin{array}{l}14 \\
22 \\
88\end{array}$ \\
\hline $\begin{array}{l}\text { Propyl- } \\
\text { urethan }\end{array}$ & $\begin{array}{l}1,0 \\
2,0\end{array}$ & $\begin{array}{l}44 \\
94\end{array}$ \\
\hline $\begin{array}{l}\text { Phenyl- } \\
\text { urethan }\end{array}$ & $\begin{array}{l}0,025 \\
0,05 \\
0,1\end{array}$ & $\begin{array}{l}33 \\
55 \\
90\end{array}$ \\
\hline
\end{tabular}

Wahl des Substrats, der Oxalsäure, und ihrer Konzentration bedingt. Auch für die Wirkung der Narkotika auf chemische Vorgänge in Zellen wurde ja gezeigt, dass die besondere Natur der chemischen Reaktion auf die Wirkungsstärken nur von geringem Einfluss ist; beispielsweise wurden Oxydationsgeschwindigkeit und Gärungsgeschwindigkeit in der Hefezelle durch sehr ähnliche Konzentrationen indifferenter Narkotika gehemmt ${ }^{2}$ ).

Aus einem Vergleich der beiden Tabellen geht endlich hervor, dass die Wirkung der Narkotika auf das Modell sich in einem sehr wesentlichen Punkt von der Wirkung auf die Zelloxydationen unterscheidet. Die Wirkung der Narkotika auf die Oxydationsgeschwindigkeit in Zellen wächst viel schneller mit der Konzentration als die Wirkung auf die Modelloxydatiousgeschwindigkeit. Dieser Unterschied dürfte damit zusammenhängen, dass einerseits die Adsorption der Narkotika an

1) Siehe 0. Warburg, in Asher-Spiro, 1. c.

2) O. Warburg, in Asher-Spiro, 1. c. 
den gequollenen Gelen der Zelle nach einem anderen Gesetz verläuft als die Adsorption an der Kohleoberfläche [die Adsorptionsisothermen sind weniger gekrümmt ${ }^{1}$ )]; dass anderseits bei der Narkotikawirkang in der Zelle nicht nur die Adsorption an den Gelen, sondern auch an den Ultramikronen der Sole eine Rolle spielt. Das Modell wäre ähnlicher, wenn wir die Oxalsäure an der Oberfläche eines gequollenen Gels unter der Einwirkung eines im Solzustand befindlichen Katalysators verbrennen würden.

\section{Bemerkung iiber den Mechanismus der Kohlekatalyse und über den Mechanismus der Hemmungen.}

Dass die Verbrennung der Oxalsäure an der Kohleoberfläche vor sich geht, dürfte kaum einem $/$ weifel unterliegen; wir haben es hier mit einem typischen Fall von Oberflächenkatalyse zu tun. Wir wissen jedoch nicht, in welcher Weise die an der Kohleoberfläche herrschenden Bedingungen die Oxydationsbeschleunigung herbeiführen, und es sei daran erinnert, dass viele Stoffe, die sonst nicht beständiger sind wie Oxalsäure, an der Kohle zwar verdichtet, aber nicht verbrannt werden. So wird Zucker bekanntlich adsorbiert, aber nicht chemisch ${ }^{2}$ ) verändert.

Auf eine Tatsache möchte ich in diesem Zusammenhang hinweisen. Die Blutkohle von Merck, die in meinen Versuchen verwendet wurde und die auch früher, dank ihres starken Adsorptionsvermögens, vielfach zu Adsorptionsversuchen verwendet wurde, ist zwar mit Säuren gereinigt, enthält aber nichtsdestoweniger reichlich Mineralbestandteile ${ }^{3}$ ), darunter auch Eisen. Man kann sich davon leicht überzeugen, wenn man die Kohle an der Luft glüht. Es bleibt dann eine helle Asche, die starke Eisenreaktion gibt. Verascht man nicht, sondern kocht die Kohle mit Säure aus, so gehen nur Eisenspuren ins Filtrat.

Blutkohle ist also keineswegs reiner Kohlenstoff, sondern eine Kombination von Kohlenstoff mit Mineralbestandteilen. Gerade der Gehalt an Eisen ist, im Zusammenhang mit den oxydationsbeschleunigenden Eigenschaften der Kohle, jedenfalls beachtenswert.

1) Vgl. die Werte für Thymol in Asher-Spiro, l. c.

2) Rona und Michaelis, Biochem. Zeitschr. Bd. 16 S. 489.1909.

3) Aschebestimmungen bei Glassner und Suida. Liebig's Annalen Bd. 357 S. 95.1907. 
Was den Mechanismus der Hemmungen anbetrifft, so ist das nächstliegende, an eine Verdrängung der Oxalsäure von dèr Kohleoberfläche zu denken; in der Tat wird aus einem Gemisch zweier adsorbierbarer Substanzen jede Komponente stets schwächer adsorbiert als aus reiner Lösung ${ }^{1}$ ). Stärker adsorbierbare Substanzên wirken stärker verdrängend ${ }^{1}$ ), die Reihenfolge der Wirkungsstärkẹu am Modell wäre dann so zu erklären, dass um so mehr Oxalsäure von der Oberfläche verdrängt wird, je kapillaraktiver das betreffende Narkotikum ist.

Soll auch nicht in Abrede gestellt werden, dass die Adsorptionsverdrängung als ursächliches Moment der Hemmungswirkungen in Betracht kommt, so halte ich es doch für sehr fraglicì, ob sie allein in Betracht kommt. Ich vermute vielmehr, dass a $\mathrm{uch} \mathrm{Ver}$ änderungen des Milieus an der Kohleoberfläche, die nicht auf Adsorptionsverdrängu ug beruhen, eine wesentliche Rolle spielen. Ist diese Hypothese richtig, so wäre zu erwarten, dass ein Narkotikum auf die verschiedensten Oberflächenkatalysen, bei ganz verschiedenen Substraten, ähnlich wirkt.

\section{Gasanalytische Methodik.}

Sauerstoffverbrauch und Kohlensäureproduktion wurden nach einer von Siebeck und dem Verfasser ausgearbeiteten Methode gemessen. Die Methode ist von Siebeck in Abderhalden's Biochemischen Arbeitsmethoden ${ }^{2}$ ) genauer beschrieben, und so kann ich mich hier mit einigen kurzen Bemerkungen begnügen. Das Volumen der Schüttelgefässe, in denen die Druckverminderungen auftraten, betrug etwa $11 \mathrm{ccm}$ (nach Einfüllen der 1,5 ccm Kohlesuspension und der zur Absorption der Kohlensäure dienenden Kalilauge). Ein Ausschlag am Manometer um $1 \mathrm{~mm}$ entsprach unter diesen Bedingungen ungefähr einem Sauerstoffverbrauch von $1 \mathrm{cmm}$. Da der Fehler nicht mehr als $2 \mathrm{~mm}$ beträgt, so sind also die Angaben genau auf $2 \mathrm{im}$ Verhältnis zur Zahl der verbrauchten Kubikmillimeter.

Sollten Sauerstoffverbrauch und Kohlensäureproduktion gemessen werden, so wurden in zwei Schüttelgefässe je $1,5 \mathrm{ccm}$ der gleichen Kohlesuspension eingefüllt. In e in Schüttelgefäss wurde, wie gewöhnlich, in den Einsatz Kalilauge gegeben; in das andere wurde keine Kalilange gegeben. In dem einen Schüttelgefäss tritt dann ein positiver, in dem anderen ein negativer Druck auf. Der negative Druck entspricht dem Sauerstoffverbrauch, der positive, vermehrt um den in dem

1) Michaelis und Rona, Biochem. Zeitschr. Bd. 15 S. $209.1908 .-$ Freundlich und Masius in Freundlich, Kapillarchemie S. 163. Leipzig 1909.

2) Der betreffende Band befindet sich im Druck, 
560 Otto Warburg: Über Verbrennung der Oxalsäure an Blutkohle etc.

anderen Gläschen auftretenden negativen, der an den Gasraum abgegebenen Kohlensäure. $\mathrm{Zu}$ der an den Gasraum abgegebenen Kohlensäure ist die in der Flüssigkeit gelöste Kohlensäure zu addieren; dieses Korrektionsglied ergibt sich aus dem Partialdruck der Kohlensäure in dem Gefäss, der Flüssigkeitsmenge und dem Absorptionskoeffizienten der Kohlensäure bei der Versuchstemperatur. Die Adsorption der Kohlensäure an der Kohle ist ein Korrektionsglied, das nicht berechnet werden konnte. Diese Adsorption wird dahin wirken; dass der Wert für die Kohlensäure etwas zu klein ausfällt. 